\title{
Chemical Constituents from Rhizomes of Curculigo capitulata
}

\author{
Lei Di, Kai-Jin Wang, Cui-Cui Zhu, and Ning Li*
}

\begin{abstract}
Anhui Province Key Laboratory of Research and Development of Chinese Medicine, School of Life Sciences, Anhui University, Hefei 230039, P. R. China. *E-mail: wkjahla@sina.com (K. J. W.),ln0110@sina.com (N. L.) Received June 29, 2010, Accepted August 12, 2010
\end{abstract}

Key Words: Curculigo capitulata, Hypoxidaceae, Constituents, Capituloside B

The herb Curculigo capitulata (Lour.) Ktze is widely distributed in Southern and Southwestern China. It has been used as a tonic and a medicine for the treatment of dysmenorrhea and rheumatism. ${ }^{1}$ This species has been reported to be rich in phenolic compounds and norlignan compounds with skeletons $\mathrm{Ph}-\mathrm{C}_{5}-\mathrm{Ph}$, which have been found to possess some beneficial pharmacological effects, including anti-arrhythmic properties, antioxidant activity, and vasoconstrictor activity. ${ }^{2-6}$

Previously, we reported the chemical constituents from the rhizomes of $C$. capitulata collected in xishuangbanna region of Yunnan Province, China. ${ }^{3,7}$ To compare the chemical constituents' differences of the same Curculigo species belonging to different geographical distribution and climatic conditions, we investigated the rhizomes of C. capitulata collected in Napo region of Guangxi Province, China. Recently, we reported two novel norlignan derivatives with the rearranged skeletons, named as crassifoside I and sinensigenin C, from this species collected in Napo region of Guangxi Province, China. ${ }^{8}$ To further search more novel compounds, the minor constituents of this plant were investigated. This paper deals with the isolation and structure elucidation of one novel norlignan derivative, named as capituloside B (1), together with eight known compounds, curcapicycloside (2), ${ }^{4}$ capituloside (3), ${ }^{3}$ breviscaside B (4), ${ }^{9}$ crassifogenin C (5), ${ }^{10}$ breviscapin A(6), ${ }^{11}$ methyl-4-O-coumaroylquinate (7), ${ }^{12}$
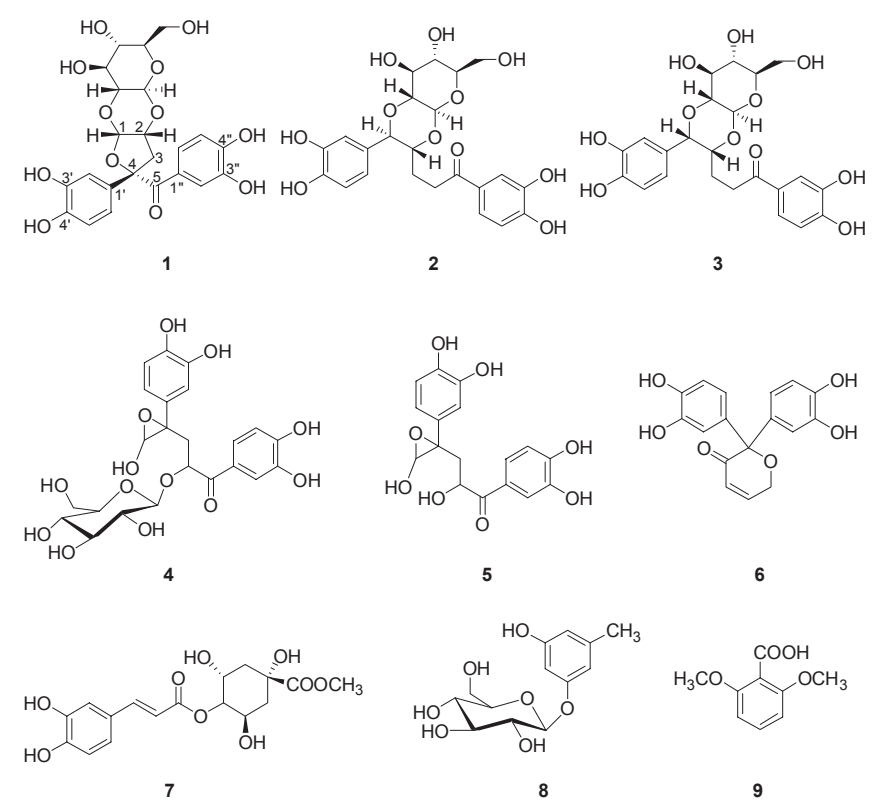

Figure 1. Structures of compounds 1-9. orcinol glucoside (8) ${ }^{13,14}$ and 2,6-dimethoxy-benzioc acid (9), ${ }^{2}$ from its rhizomes as shown in Figure 1. Their structures were established by spectroscopic analysis, especially using 2DNMR techniques $\left({ }^{1} \mathrm{H}-{ }^{1} \mathrm{H}\right.$ COSY, HMQC, HMBC, and NOESY) and comparisons of their data with literature values. Compounds 4-7 were isolated for the first time from this plant, and compound 7 was the first example isolated from the family.

\section{Experimental}

General experimental procedures. Optical rotation was measured on a Horiba SEPA-300 polarimeter. A UV-2401PC spectrometer was used to obtain the UV spectrum in methanol (MeOH). IR spectra were recorded on Nexus 870-FT-IR spectrophotometer with potassium bromide $(\mathrm{KBr})$ pellets. NMR spectra were measured on a Bruker AM-400 spectrometer with TMS as an internal standard. FAB-MS and HR-TOF-MS were performed on a VG Autospec-3000 spectrometer and API-QSTARPulsar-1 spectrometer, respectively. Column chromatography was carried out on Sephadex LH-20 gel (25 - $100 \mu \mathrm{m}$, Pharmacia Fine Chemical Co. Ltd.) and Chromatorex ODS (30 - $50 \mu \mathrm{m}$, Fuji Silysia Chemical Co. Ltd.). Thin layer chromatography (TLC) was carried out on silica gel G precoated plates (Qingdao Haiyang Chemical Co. Ltd.), and spots were detected by spraying with $5 \% \mathrm{H}_{2} \mathrm{SO}_{4}$ in $\mathrm{EtOH}$ followed by heating.

Plant material. The rhizomes of $C$. capitulata were collected in Napo, Guangxi Province, China, in August 2007 and identified by Prof. Dr. Kai-Jin Wang from the School of Life Sciences, Anhui University, where a voucher specimen (No. 20070803) was deposited.

Extraction and isolation. The air-dried and powdered rhizomes of C. capitulata $(1.25 \mathrm{~kg})$ were extracted with $85 \% \mathrm{EtOH}$ $(3 \times 6 \mathrm{~L})$ under reflux for $3 \mathrm{~h}$. The combined organic layer was concentrated in vacuo to achieve a residue $(55 \mathrm{~g})$. The residue was suspended in $\mathrm{H}_{2} \mathrm{O}$ and then passed through a D101 resin column eluting sequentially with water followed by $30 \%, 60 \%$, and $90 \%$ aqueous $\mathrm{MeOH}$. The fraction $(5.3 \mathrm{~g})$ eluted from $30 \% \mathrm{MeOH}$ was purified by Sephadex LH-20 chromatography $\left(\mathrm{MeOH}-\mathrm{H}_{2} \mathrm{O}, 0: 1-1: 0\right)$ to yield two fractions $\left(\mathrm{A}_{1}\right.$ and $\left.\mathrm{A}_{2}\right)$. Fraction $\mathrm{A}_{1}$ was subjected to further separation on Sephadex LH-20 chromatography (EtOH-acetone, 1:1) and ODS $\left(\right.$ EtOH- $\mathrm{H}_{2} \mathrm{O}$, 0:1-1:0) to afford $4(17 \mathrm{mg})$ and 5 (8 mg). Fraction $A_{2}$ was purified by Sephadex LH-20 chromatography $(\mathrm{EtOH})$ and then ODS (EtOH- $\left.\mathrm{H}_{2} \mathrm{O}, 0: 1-1: 0\right)$ to yield 7 (15 mg) and 8 (66 mg). The fraction $(5.8 \mathrm{~g})$ eluted from $60 \% \mathrm{MeOH}$ was purified by Sephadex LH-20 chromatography $\left(\mathrm{MeOH}-\mathrm{H}_{2} \mathrm{O}, 0: 1-1: 0\right)$ to 
yield three fractions $\left(\mathrm{B}_{1}-\mathrm{B}_{3}\right)$. Fraction $\mathrm{B}_{1}$ was subjected on ODS $\left(\mathrm{MeOH}-\mathrm{H}_{2} \mathrm{O}, 0: 1-1: 0\right)$ and then Sephadex LH-20 (EtOH) to afford compounds $\mathbf{1}(23 \mathrm{mg}$ ) and $\mathbf{6}(6 \mathrm{mg})$. Compounds 2 (5 mg) and $3(9 \mathrm{mg})$ was obtained from fraction $\mathrm{B}_{3}$ by column chromatography on Sephadex LH-20 (EtOH-acetone, 1:1) and then ODS (MeOH- $\left.\mathrm{H}_{2} \mathrm{O}, 0: 1-1: 0\right)$. The fraction (3.8 g) eluted from $90 \% \mathrm{MeOH}$ was subjected to chromatography on Sephadex LH-20 (MeOH- $\mathrm{H}_{2} \mathrm{O}, 0: 1-1: 0$, then EtOH) to yield compound 9 (21 mg).

Acidic hydrolysis of compound 1. Compound 1 (23 mg) was refluxed with $2 \mathrm{~mol} \mathrm{~L}^{-1} \mathrm{HBr}$-dioxane $(1: 1, \mathrm{v} / \mathrm{v}, 2 \mathrm{~mL})$ on a water bath for $6 \mathrm{~h}$. The reaction mixture was evaporated to dryness. The dry reaction mixture was extracted with $\mathrm{CHCl}_{3}$ and $\mathrm{H}_{2} \mathrm{O}$ four times. The $\mathrm{H}_{2} \mathrm{O}$-souble fraction was evaporated to dryness. The dried sugar residue was diluted in $1 \mathrm{~mL}$ pyridine without water and treated with $0.5 \mathrm{~mL}$ trimethyl-chlorsilan (TMCS) and stirred at $60{ }^{\circ} \mathrm{C}$ for $5 \mathrm{~min}$. After drying the solution with a stream of $\mathrm{N}_{2}$, the residue was extracted with ether $(1 \mathrm{~mL})$. The ether layer was analyzed by $\mathrm{GC}$ with the following conditions: HP AC-5 quartz capillary column $(30 \mathrm{~m} \times 0.32 \mathrm{~mm})$; detector: FID $\left(250{ }^{\circ} \mathrm{C}\right)$; injection temperature: $250{ }^{\circ} \mathrm{C}$; column temperature: $180-280{ }^{\circ} \mathrm{C}$; rate: $3{ }^{\circ} \mathrm{C} / \mathrm{min}$; and retention times (min): the derivative of D-glucose (7.22).

Capituloside B (1): White powder. $[\alpha]_{\mathrm{D}}^{21}=-82.1(c=0.12$, $\mathrm{MeOH})$; IR (KBr) $v_{\max } \mathrm{cm}^{-1}: 3419(\mathrm{OH}), 2924,1665(\mathrm{C}=\mathrm{O})$, 1600, 1522, 1440, 1375, 1294, 1201, 1107, 1032, 872, 808, 784; UV (MeOH) $\lambda_{\max } \mathrm{nm}(\log \varepsilon): 204$ (4.35), 232 (3.97), 284 (3.79), 314 (3.69). ${ }^{1} \mathrm{H}$ - and ${ }^{13} \mathrm{C}-\mathrm{NMR}$ data see Table 1. HR-TOFMS (negative mode): $m / z$ 491.1178 [M-H] ${ }^{-}$(calcd. 491.1189 for $\mathrm{C}_{23} \mathrm{H}_{23} \mathrm{O}_{12}$ ).

Curcapicycloside (2): White powder. ${ }^{1} \mathrm{H}-\mathrm{NMR}(400 \mathrm{MHz}$, $\left.\mathrm{CD}_{3} \mathrm{OD}\right) \delta 4.61(1 \mathrm{H}, \mathrm{d}, J=6.0 \mathrm{~Hz}, \mathrm{H}-1), 4.54(1 \mathrm{H}, \mathrm{m}, \mathrm{H}-2)$, 2.07 (1H, m, H-3a), 1.86 (1H, m, H-3b), 3.07 (2H, m, H-4), $6.98\left(1 \mathrm{H}, \mathrm{d}, J=1.8 \mathrm{~Hz}, \mathrm{H}-2^{\prime}\right), 6.80\left(1 \mathrm{H}, \mathrm{d}, J=8.2 \mathrm{~Hz}, \mathrm{H}-5^{\prime}\right)$, $6.86\left(1 \mathrm{H}, \mathrm{dd}, J=8.1,2.0 \mathrm{~Hz}, \mathrm{H}-6^{\prime}\right), 7.44(1 \mathrm{H}, \mathrm{d}, J=2.0 \mathrm{~Hz}$, H-2"), 6.85 (1H, d, $J=8.8 \mathrm{~Hz}, \mathrm{H}-5 "), 7.44$ (1H, dd, $J=8.7,2.0$ Hz, H-6"), 4.84 (1H, d, J=8.5 Hz, H-1"'), 3.44 (1H, m, H-2"'), 3.66 (1H, m, H-3'"), 3.54 (1H, m, H-4"'), 3.35 (1H, m, H-5"'), $3.91\left(1 \mathrm{H}, \mathrm{dd}, J=12.0,2.0 \mathrm{~Hz}, \mathrm{Ha}-6^{\prime \prime \prime}\right), 3.74$ (1H, dd, $J=12.0$, $\left.5.4 \mathrm{~Hz}, \mathrm{Hb}-6{ }^{\prime \prime \prime}\right) ;{ }^{13} \mathrm{C}-\mathrm{NMR}\left(100 \mathrm{MHz}, \mathrm{CD}_{3} \mathrm{OD}\right) \delta 79.5$ (d, C-1), 79.9 (d, C-2), 27.6 (t, C-3), 34.7 (t, C-4), 200.9 (s, C-5), 131.9 (s, C-1'), 116.2 (d, C-2'), 146.4 (s, C-3'), 146.4 (s, C-4'), 116.2 (d, C-5'), 120.8 (s, C-6'), 130.5 (s, C-1"), 116.0 (s, C-2"), 146.5 (s, C-3"), 152.1 (s, C-4"), 115.8 (d, C-5"), 123.1 (d, C-6"), 97.4 (d, C-1"'), 73.9 (d, C-2"'), 76.0 (d, C-3"'), 71.9 (d, C-4"'), 75.6 (d, C-5"'), 62.6 (t, C-6"'); FAB-MS (+) $m / z 479$ [M+H] $]^{+}$.

Capituloside (3): White powder, ${ }^{1} \mathrm{H}-\mathrm{NMR}\left(400 \mathrm{MHz}, \mathrm{CD}_{3}\right.$ OD) $\delta 4.92$ (1H, d, $J=3.6 \mathrm{~Hz}, \mathrm{H}-1), 4.20$ (1H, m, H-2), 2.19 (1H, m, Ha-3), 1.53 (1H, m, Hb-3), $2.91(2 \mathrm{H}, \mathrm{m}, \mathrm{H}-4), 6.88(1 \mathrm{H}$, d, $\left.J=2.0 \mathrm{~Hz}, \mathrm{H}-2^{\prime}\right), 6.79\left(1 \mathrm{H}, \mathrm{d}, J=8.4 \mathrm{~Hz}, \mathrm{H}-5{ }^{\prime}\right), 6.73(1 \mathrm{H}$, dd, $J=8.4,2.0 \mathrm{~Hz}, \mathrm{H}-6 '), 7.39$ (1H, d, $\left.\left.J=2.0 \mathrm{~Hz}, \mathrm{H}-2^{\prime \prime}\right)\right), 6.83$ $(1 \mathrm{H}, \mathrm{d}, J=8.0 \mathrm{~Hz}, \mathrm{H}-5 "), 7.38$ (1H, dd, $J=8.0,2.0 \mathrm{~Hz}, \mathrm{H}-6 ")$, $4.64\left(1 \mathrm{H}, \mathrm{d}, J=7.6 \mathrm{~Hz}, \mathrm{H}-1^{\prime \prime \prime}\right), 3.23(1 \mathrm{H}, \mathrm{dd}, J=9.6,8.4 \mathrm{~Hz}$, H-2"'), 3.69 (1H, m, H-3"'), 3.43 (1H, m, H-4"'), 3.49 (1H, m, H-5"'), 3.93 (1H, dd, $J=12.0,2.0 \mathrm{~Hz}, \mathrm{Ha}-6 " '), 3.75$ (1H, dd, $J=$ 12.0, $5.2 \mathrm{~Hz}, \mathrm{Hb}-6$ "'); ${ }^{13} \mathrm{C}-\mathrm{NMR}\left(100 \mathrm{MHz}, \mathrm{CD}_{3} \mathrm{OD}\right) \delta 80.4(\mathrm{~d}$, C-1), 79.2 (d, C-2), 21.3 (t, C-3), 34.7 (t, C-4), 201.1 (s, C-5), 130.5 (s, C-1'), 115.7 (d, C-2'), 146.3 (s, C-3'), 145.7 (s, C-4'),
Table 1. ${ }^{1} \mathrm{H}-\mathrm{NMR}$ and ${ }^{13} \mathrm{C}-\mathrm{NMR}$ data of $1\left(400 \mathrm{MHz}\right.$, in $\mathrm{CD}_{3} \mathrm{OD}, \delta$ ppm, $J$ in $\mathrm{Hz}$ )

\begin{tabular}{|c|c|c|c|}
\hline position & $\delta(\mathrm{C})$ & $\delta(\mathrm{H})$ & $\operatorname{HMBC}(\mathrm{H} \rightarrow \mathrm{C})$ \\
\hline 1 & $98.5(d)$ & \multirow{3}{*}{$\begin{array}{l}5.46(d, J=3.7) \\
4.60(t d, J=9.2,3.6) \\
3.56(d d, J=12.9,9.7) \\
2.11(d d, J=13.0,8.7)\end{array}$} & C-3, C-4, Glc.C-2 \\
\hline 2 & $77.1(d)$ & & C-3, C-4, Glc.C-1 \\
\hline 3 & $36.6(t)$ & & C-1, C-2, C-4, C-5 \\
\hline 4 & $93.6(s)$ & & \\
\hline 5 & $198.0(s)$ & & \\
\hline $1^{\prime}$ & $135.5(s)$ & & \\
\hline $2^{\prime}$ & $112.7(d)$ & $6.82(d, J=1.6)$ & C-4, C-1', C-3' \\
\hline $3^{\prime}$ & $146.1(s)$ & & \\
\hline $4^{\prime}$ & $146.8(s)$ & & \\
\hline $5^{\prime}$ & $116.73(d)^{a}$ & $6.74(d, J=8.4)$ & C-4, C-1', C-3' \\
\hline $6^{\prime}$ & $116.71(d)^{a}$ & $6.71(d d, J=8.4,1.6)$ & C-4, C-1', C-2', C-4' \\
\hline $1 "$ & $127.9(s)$ & & \\
\hline $2^{\prime \prime}$ & $119.3(d)$ & $7.48(d, J=2.0)$ & C-5, C-3", C-4", C-6" \\
\hline $3 "$ & $145.6(s)$ & & \\
\hline $4 "$ & $151.6(s)$ & & \\
\hline $5^{\prime \prime}$ & $115.2(d)$ & $6.64(d, J=8.4)$ & C-5, C-1", C-3", C-4" \\
\hline 6" & $126.2(d)$ & $7.43(d d, J=8.4,2.0)$ & C-5, C-2", C-4" \\
\hline \multicolumn{4}{|l|}{ Glc. } \\
\hline 1 & $94.1(d)$ & $4.65(d, J=7.9)$ & $\mathrm{C}-2$ \\
\hline 2 & $77.4(d)$ & $3.06(d d, J=9.1,8.2)$ & $\mathrm{C}-1$ \\
\hline 3 & $74.7(d)$ & $3.40(m)$ & \\
\hline 4 & $71.8(d)$ & $3.32(\mathrm{~m})$ & \\
\hline 5 & $79.6(d)$ & $3.35(m)$ & \\
\hline \multirow[t]{2}{*}{6} & $62.6(t)$ & $3.86(d d, J=12.0,1.8)$ & \\
\hline & & $3.68(d d, J=12.0,5.4)$ & \\
\hline
\end{tabular}

${ }^{\bar{a}}$ Values may be interchangeable.

116.2 (d, C-5'), 118.3 (s, C-6'), 130.4 (s, C-1"), 114.2 (s, C-2"), 146.3 (s, C-3"), 152.0 (s, C-4"), 115.9 (d, C-5"), 123.0 (d, C-6"), 93.9 (d, C-1"'), 81.9 (d, C-2"'), 75.0 (d, C-3'"), 72.1 (d, C-4"'), 79.9 (d, C-5"'), 62.6 (t, C-6"'); FAB-MS (+) m/z 479 [M+H] $]^{+}$.

Breviscaside B (4): White powder, ${ }^{1} \mathrm{H}-\mathrm{NMR}(400 \mathrm{MHz}$, $\left.\mathrm{CD}_{3} \mathrm{OD}\right) \delta 5.56(1 \mathrm{H}, \mathrm{s}, \mathrm{H}-1), 2.05(1 \mathrm{H}, \mathrm{dd}, J=13.4,3.0 \mathrm{~Hz}$, Ha-3), 3.57 (1H, dd, $J=14.0,6.4 \mathrm{~Hz}, \mathrm{Hb}-3), 4.21$ (1H, m, H-4), $7.01\left(1 \mathrm{H}, \mathrm{d}, J=2.0 \mathrm{~Hz}, \mathrm{H}-2^{\prime}\right), 6.71\left(1 \mathrm{H}, \mathrm{d}, J=8.4 \mathrm{~Hz}, \mathrm{H}-5^{\prime}\right), 6.87$ $\left(1 \mathrm{H}, \mathrm{dd}, J=8.4,2.0 \mathrm{~Hz}, \mathrm{H}-6^{\prime}\right), 7.52\left(1 \mathrm{H}, \mathrm{d}, J=2.0 \mathrm{~Hz}, \mathrm{H}-2^{\prime \prime}\right)$, $6.65(1 \mathrm{H}, \mathrm{d}, J=8.4 \mathrm{~Hz}, \mathrm{H}-5 "), 7.46(1 \mathrm{H}, \mathrm{dd}, J=8.4,2.0 \mathrm{~Hz}$, H-6"), 4.30 (1H, d, J=7.6 Hz, H-1"'), 3.13-3.39 (4H, m, Glc.H), 3.87 (1H, dd, $J=12.0,2.0$ Hz, Ha-6'"), 3.70 (1H, dd, $J=12.4$, $5.2 \mathrm{~Hz}, \mathrm{Hb}-6 " ') ;{ }^{13} \mathrm{C}-\mathrm{NMR}\left(100 \mathrm{MHz}, \mathrm{CD}_{3} \mathrm{OD}\right) \delta 104.0$ (d, C-1), 94.7 (s, C-2), 43.1 (t, C-3), 86.0 (d, C-4), 200.6 (s, C-5), 136.1 (s, C-1'), 113.3 (d, C-2'), 145.7 (s, C-3'), 146.4 (s, C-4'), 116.4 (d, C-5'), 117.3 (d, C-6'), 128.2 (s, C-1"), 119.4 (d, C-2"), 145.5 (s, C-3"), 151.1 (s, C-4"), 115.1 (d, C-5"), 126.2 (d, C-6"), 103.7 (d, C-1"'), 74.9 (d, C-2'"), 77.8 (d, C-3"'), 71.4 (d, C-4"'), 77.9 (d, C-5"'), 62.6 (t, C-6"'); FAB-MS (-) m/z 509 [M-H] ${ }^{-}$.

Crassifogenin C (5): White powder, ${ }^{1} \mathrm{H}-\mathrm{NMR}(400 \mathrm{MHz}$, $\left.\mathrm{CD}_{3} \mathrm{OD}\right) \delta 5.42(1 \mathrm{H}, \mathrm{s}, \mathrm{H}-1), 1.84(1 \mathrm{H}, \mathrm{dd}, J=13.7,2.8 \mathrm{~Hz}$, Ha-3), 3.49 (1H, dd, $J=6.2,13.7 \mathrm{~Hz}, \mathrm{Hb}-3), 4.08$ (1H, m, H-4), $6.93\left(1 \mathrm{H}, \mathrm{d}, J=1.9 \mathrm{~Hz}, \mathrm{H}-2^{\prime}\right), 6.67\left(1 \mathrm{H}, \mathrm{d}, J=8.3 \mathrm{~Hz}, \mathrm{H}-5^{\prime}\right)$, $6.75\left(1 \mathrm{H}, \mathrm{dd}, J=8.3,2.0 \mathrm{~Hz}, \mathrm{H}-6{ }^{\prime}\right), 7.49(1 \mathrm{H}, \mathrm{d}, J=1.9 \mathrm{~Hz}$, H-2"), 6.62 (1H, d, $J=8.4 \mathrm{~Hz}, \mathrm{H}-5 "), 7.45$ (1H, dd, $J=8.4,1.9$ $\mathrm{Hz}, \mathrm{H}-6 ") ;{ }^{13} \mathrm{C}-\mathrm{NMR}\left(100 \mathrm{MHz}, \mathrm{CD}_{3} \mathrm{OD}\right) \delta 105.5$ (d, C-1), 93.3 (s, C-2), 45.5 (t, C-3), 77.8 (d, C-4), 200.4 (s, C-5), 136.7 
(s, C-1'), 113.3 (d, C-2'), 145.7 (s, C-3'), 146.5 (s, C-4'), 116.5 (d, C-5'), 117.3 (s, C-6'), 128.7 (s, C-1"), 119.6 (s, C-2"), 145.5 (s, C-3"), 151.2 (s, C-4"), 115.2 (d, C-5"), 126.4 (d, C-6"); FAB-MS (-) $m / z 347$ [M-H].

Breviscapin A (6): Black powder, ${ }^{1} \mathrm{H}-\mathrm{NMR}(400 \mathrm{MHz}$, acetone- $\left.d_{6}\right) \delta 6.14(1 \mathrm{H}, \mathrm{dt}, J=10.4,2.2 \mathrm{~Hz}, \mathrm{H}-4), 7.13(1 \mathrm{H}, \mathrm{dt}, J=$ $10.4,2.8 \mathrm{~Hz}, \mathrm{H}-5), 4.34$ (1H, dd, $J=2.8,2.3 \mathrm{~Hz}, \mathrm{H}-6), 6.72$ $\left(1 \mathrm{H}, \mathrm{d}, J=2.0 \mathrm{~Hz}, \mathrm{H}-2^{\prime}\right), 6.76\left(1 \mathrm{H}, \mathrm{d}, J=8.0 \mathrm{~Hz}, \mathrm{H}-5{ }^{\prime}\right), 6.60$ $(1 \mathrm{H}, \mathrm{dd}, J=8.0,2.0 \mathrm{~Hz}, \mathrm{H}-6 '), 6.72(1 \mathrm{H}, \mathrm{d}, J=2.0 \mathrm{~Hz}, \mathrm{H}-2 ")$, $6.76(1 \mathrm{H}, \mathrm{d}, J=8.0 \mathrm{~Hz}, \mathrm{H}-5 "), 6.60(1 \mathrm{H}, \mathrm{dd}, J=8.0,2.0 \mathrm{~Hz}$, H-6"); ${ }^{13} \mathrm{C}-\mathrm{NMR}\left(100 \mathrm{MHz}\right.$, acetone- $\left.d_{6}\right) \delta 88.3(\mathrm{~s}, \mathrm{C}-2), 196.2$ (s, C-3), 128.1 (d, C-4), 150.2 (d, C-5), 62.8 (t, C-6), 133.5 (s, C-1'), 117.3 (d, C-2'), 146.2 (s, C-3'), 146.6 (s, C-4'), 116.2 (d, C-5'), 121.3 (d, C-6'), 133.5 (s, C-1"), 117.3 (d, C-2"), 146.2 (s, C-3"), 146.6 (s, C-4"), 116.2 (d, C-5"), 121.3 (d, C-6"); FAB-MS (-) $m / z 313[\mathrm{M}-\mathrm{H}]^{-}$.

Methyl-4-O-coumaroylquinate (7): White powder, ${ }^{1} \mathrm{H}-\mathrm{NMR}$ $\left(400 \mathrm{MHz}, \mathrm{CD}_{3} \mathrm{OD}\right) \delta 2.19(1 \mathrm{H}, \mathrm{m}, \mathrm{Ha}-2), 2.04(1 \mathrm{H}, \mathrm{m}, \mathrm{Hb}-2)$, 4.27 (1H, m, H-3), 4.83 (1H, dd, $J=8.8,2.7 \mathrm{~Hz}, \mathrm{H}-4), 4.29$ (H, m, H-5), 2.20 (1H, m, Ha-6), $2.06(1 \mathrm{H}, \mathrm{m}, \mathrm{Hb}-6), 3.76(3 \mathrm{H}, \mathrm{s}$, H-8), $7.08\left(1 \mathrm{H}, \mathrm{d}, J=2.0 \mathrm{~Hz}, \mathrm{H}-2^{\prime}\right), 6.79(1 \mathrm{H}, \mathrm{d}, J=8.2 \mathrm{~Hz}$, H-5'), 6.98 (1H, dd, $J=8.0,2.0 \mathrm{~Hz}, \mathrm{H}-6$ '), 7.64 (1H, d, $J=15.9$ $\left.\mathrm{Hz}, \mathrm{H}^{-} 7^{\prime}\right), 6.73\left(1 \mathrm{H}, \mathrm{d}, J=15.9 \mathrm{~Hz}, \mathrm{H}-8^{\prime}\right) ;{ }^{13} \mathrm{C}-\mathrm{NMR}(100 \mathrm{MHz}$, $\left.\mathrm{CD}_{3} \mathrm{OD}\right) \delta 76.5$ (s, C-1), 42.2 (t, C-2), 65.8 (d, C-3), 78.6 (d, C-4), 69.1 (d, C-5), 38.5 (t, C-6), 175.7 (s, C-7), 53.0 (q, C-8), 127.9 (s, C-1'), 115.2 (d, C-2'), 149.6 (s, C-3'), 146.9 (s, C-4'), 116.5 (d, C-5'), 123.0 (d, C-6'), 147.2 (d, C-7'), 115.4 (d, C-8'), 169.0 (s, C-9'); FAB-MS (-) m/z 365 [M-H].

Orcinol glucoside (8): Colorless needle $(\mathrm{MeOH}),{ }^{1} \mathrm{H}-\mathrm{NMR}$ (400 MHz, CD 3 OD) $\delta 2.11\left(3 \mathrm{H}, \mathrm{s}, \mathrm{CH}_{3}\right), 6.31(1 \mathrm{H}, \mathrm{s}, \mathrm{H}-2), 6.27$ (1H, s, H-4), 6.19(1H, s, H-6), 4.74 (1H, d, J=7.6 Hz, H-1'), 3.20-3.36 (4H, m, Glc H), $3.61(1 \mathrm{H}, \mathrm{dd}, J=12.08,4.80 \mathrm{~Hz}$, Ha-6'), 3.79 (1H, dd, $J=12.08,1.48 \mathrm{~Hz}, \mathrm{Hb}-6$ '), 10.09 (1H, br. s, $\mathrm{C}-3-\mathrm{OH}) ;{ }^{13} \mathrm{C}-\mathrm{NMR}\left(100 \mathrm{MHz}, \mathrm{CD}_{3} \mathrm{OD}\right) \delta 158.7$ (s, C-1), 100.8 (d, C-2), 157.8 (s, C-3), 108.4 (d, C-4), 139.9 (s, C-5), 109.8 (d, C-6), 100.8 (d, C-1'), 73.5 (d, C-2'), 76.7 (d, C-3'), 70.0 (d, C-4'), $76.6\left(\mathrm{~d}, \mathrm{C}-5^{\prime}\right), 61.1$ (t, C-6'), 20.3 (q, $\left.\mathrm{CH}_{3}\right)$; FAB-MS (-) $\mathrm{m} / \mathrm{z} 285$ [M-H].

2,6-Dimethoxy-benzioc acid (9): Colorless needle (EtOH), ${ }^{1} \mathrm{H}-\mathrm{NMR}$ (400 MHz, CD $\left.3 \mathrm{OD}\right) \delta 3.79$ (6H, s, 2, 6-OMe), 6.67 $(2 \mathrm{H}, \mathrm{d}, J=8.4 \mathrm{~Hz}, \mathrm{H}-3,5), 7.17(1 \mathrm{H}, \mathrm{t}, J=8.4 \mathrm{~Hz}, \mathrm{H}-4) ;{ }^{13} \mathrm{C}-$ NMR (100 MHz, CD $\left.{ }_{3} \mathrm{OD}\right) \delta 115.1$ (s, C-1), 157.8 (s, C-2, 6), 104.8 (d, C-3, 5), 131.4 (d, C-4), 56.2 (q, 2, 6-OMe), 167.0 (s, $\mathrm{C}=\mathrm{O}) ; \mathrm{EI}-\mathrm{MS} m / z 182[\mathrm{M}]^{+}$.

\section{Results and Discussion}

Capituloside B (1) was obtained as white amorphous powder. Its molecular formula $\mathrm{C}_{23} \mathrm{H}_{24} \mathrm{O}_{12}$ with twelve unsaturation degrees was determined from a quasi-molecular ion peak at $\mathrm{m} / \mathrm{z}$ $491[\mathrm{M}-\mathrm{H}]^{-}$in its FAB-MS mass spectrum and the ${ }^{13} \mathrm{C}-\mathrm{NMR}$ (DEPT) spectrum, which was supported by its HR-TOF MS observed at $m / z$ 491.1178 [M-H] ${ }^{-}$(calcd. 491.1189 for $\mathrm{C}_{23} \mathrm{H}_{23} \mathrm{O}_{12}$ ). The IR spectrum indicated absorptions of $\mathrm{OH}$ groups at 3419 , a conjugated carbonyl group at 1665 and aromatic rings at 1600 , 1522 and $1440 \mathrm{~cm}^{-1}$. The conjugated carbonyl IR band was confirmed by the ${ }^{13} \mathrm{C}$-NMR signal at $\delta_{\mathrm{C}} 198.0$ (Table 1 ). The ${ }^{1} \mathrm{H}$ NMR spectrum (Table 1) exhibited signals for one methylene group at $\delta 3.56(\mathrm{dd}, J=12.9,9.7 \mathrm{~Hz}), 2.11(\mathrm{dd}, J=13.0,8.7 \mathrm{~Hz})$, and two methine protons at $\delta 5.46(\mathrm{~d}, J=3.7 \mathrm{~Hz})$, and $4.60(\mathrm{td}$, $J=9.2,3.6 \mathrm{~Hz}$ ). The ${ }^{1} \mathrm{H}$ NMR spectrum of 1 also appeared six aromatic protons, three of them were assigned to $\mathrm{H}-2^{\prime}$ at $\delta 6.82$ $(\mathrm{d}, J=1.6 \mathrm{~Hz}), \mathrm{H}-5^{\prime}$ at $\delta 6.74(\mathrm{~d}, J=8.4 \mathrm{~Hz})$, and $\mathrm{H}-6{ }^{\prime}$ at $\delta 6.71$ (dd, $J=8.4,1.6 \mathrm{~Hz}$ ), which suggested the existence of $1,3,4-$ trisubstituted benzene ring; the remaining three aromatic protons were assigned to $\mathrm{H}-2 "$ at $\delta 7.48(\mathrm{~d}, J=2.0 \mathrm{~Hz}), \mathrm{H}-5^{\prime \prime}$ at $\delta$ $6.64(\mathrm{~d}, J=8.4 \mathrm{~Hz})$, and H-6" at $\delta 7.43(\mathrm{dd}, J=8.4,2.0 \mathrm{~Hz})$ in another 1,3,4-trisubstituted benzene ring, in which H-2" and H-6" were shifted downfield due to an ortho carbonyl group (IR $v_{\mathrm{CO}} 1665 \mathrm{~cm}^{-1}$ and $\delta_{\mathrm{C}} 198.0$ ). The ${ }^{13} \mathrm{C}$ NMR (DEPT) spectrum (Table 1) showed one methylene carbon at $\delta 36.6$ (C-3), two methine carbons at $\delta 77.1(\mathrm{C}-2)$, and $98.5(\mathrm{C}-1)$, one quaternary carbon at $\delta 93.6(\mathrm{C}-4)$, a conjugated carbonyl carbon at $\delta 198.0(\mathrm{C}-5)$, four oxygen bearing aromatic carbons at $\delta 145.6$ (C-3"), 146.1 (C-3'), 146.8 (C-4'), and $151.6(\mathrm{C}-4 ")$, six aromatic $\mathrm{CH}$ at $\delta 112.7$ (C-2'), 115.2 (C-5"), 116.71 (C-6'), 116.73 (C-5'), 119.3 (C-2"), and 126.2 (C-6"), two aromatic quaternary carbons at $\delta 127.9\left(\mathrm{C}-1^{\prime \prime}\right)$, and $135.5\left(\mathrm{C}-1^{\prime}\right)$ together with six carbons of one glucosyl moiety. The ${ }^{1} \mathrm{H}$ and ${ }^{13} \mathrm{C}$ NMR spectra indicated the presence of a glucosyl moiety. The anomeric proton signal appeared as a doublet at $\delta 4.65(\mathrm{~d}, J=7.9 \mathrm{~Hz})$ suggested a $\beta$-configured glucose unit. Acid hydrolysis of 1 with 2 mol $\mathrm{L}^{-1} \mathrm{HBr}$ under refluxing produced D-glucose as sugar residue determined by $\mathrm{GC}$ analysis. Incorporating ${ }^{13} \mathrm{C} \mathrm{NMR}$ chemical shifts it showed the presence of a $\beta$-D-glucosyl unit. All the carbons of the glucosyl moiety were assigned through direct ${ }^{1} \mathrm{H}-{ }^{13} \mathrm{C}$ correlations in the HMQC spectrum and were situated between $\delta 62.6$ and 79.6 except for that at the anomeric position, which was assigned to the signal at $\delta$ 94.1.

The ${ }^{1} \mathrm{H},{ }^{1} \mathrm{H}-\mathrm{COSY}$ correlations (Figure 2 ) of $\mathrm{H}-1 / \mathrm{H}-2$, and $\mathrm{H}-2 / \mathrm{H}-3$ showed the connectivity $\mathrm{C}(1)-\mathrm{C}(2)-\mathrm{C}(3)$, which was further confirmed by HMBC correlations of $\mathrm{H}-1 / \mathrm{C}-3, \mathrm{H}-3 / \mathrm{C}-1$, $\mathrm{H}-2 / \mathrm{C}-3$, and $\mathrm{H}-3 / \mathrm{C}-2$. The HMBC correlations (Figure 2) of $\mathrm{H}-2 / \mathrm{C}-4$ and $\mathrm{H}-3 / \mathrm{C}-4$ showed the linkage of $\mathrm{C}-3$ to $\mathrm{C}-4$. The linkage of $\mathrm{C}-1$ and $\mathrm{C}-4$ to an $\mathrm{O}$-atom was established by the HMBC correlations of $\mathrm{H}-1 / \mathrm{C}-4$ and the low-field chemical shift of C-1 and C-4, at $\delta_{C}=98.5$ and 93.6, respectively (Table 1), thus, a tetrahydrofuran ring moiety was established. The benzoyl was established by the HMBC correlations H-2"/C-5, H-5"/C-5, and $\mathrm{H}-6 " / \mathrm{C}-5$. The HMBC experiments showed the long-range couplings of H-2'/C-4, H-5'/C-4, H-6'/C-4, and H-3/C-5, which suggested that the phenyl and the benzoyl were connected with $\mathrm{C}-4$, respectively. The long range ${ }^{1} \mathrm{H}-{ }^{13} \mathrm{C}$ correlations of $\mathrm{GlcH}-1 /$ C-2, H-2/GlcC-1, GlcH-2/C-1, and H-1/GlcC-2, confirmed that the fused glucosyl moiety was GlcH-1 ether-linked to $\mathrm{C}-2$ and
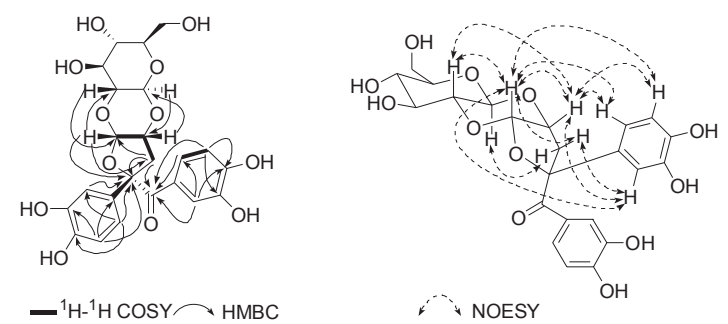

Figure 2. ${ }^{1} \mathrm{H}-{ }^{1} \mathrm{H}$ COSY, Key HMBC and NOESY correlations for $\mathbf{1}$. 
GlcH-2 to C-1.

NOESY correlations of $\mathrm{H}-1$ with $\mathrm{H}-2, \mathrm{H}-1$ and $\mathrm{H}-2$ with Glc. H-2, H-1 and H-2 with H-3a [ $\delta 2.11(d d, J=13.0,8.7)]$, and Glc. H-1 with H-3b $[\delta 3.56(d d, J=12.9,9.7)]$ as shown in Figure 2, indicated the cis relationship of H-1, H-2, H-3a and the Glc. H-2. The NOESY spectrum also exhibited crosspeaks of $\mathrm{H}-3$ a with $\mathrm{H}-2^{\prime}, \mathrm{H}-1$ and $\mathrm{H}-2$ with $\mathrm{H}-2^{\prime}, \mathrm{H}-5^{\prime}$ and $\mathrm{H}-6$ ', respectively, but not between $\mathrm{H}-1, \mathrm{H}-2$, and $\mathrm{H}-3$ a with protons of the benzoyl, indicated the cis relationship of $\mathrm{H}-1, \mathrm{H}-2, \mathrm{H}-3 \mathrm{a}$ and the phenyl, and the trans relationship of $\mathrm{H}-1, \mathrm{H}-2$, and $\mathrm{H}-3 \mathrm{a}$ with the benzoyl. Incorporating the known stereochemistry of the $\beta$-D-glucosyl unit would require $1 R, 2 S$ and $4 R$ stereochemistry in $\mathbf{1}$. Therefore, the structure of $\mathbf{1}$ was deduced as a glucosyl-fused norlignan derivative, named capituloside B (Fig. 1).

Eight known compounds, curcapicycloside (2), ${ }^{4}$ capituloside (3) ${ }^{3}$ breviscaside B (4), ${ }^{9}$ crassifogenin C (5), ${ }^{10}$ breviscapin A (6) ${ }^{11}$ methyl-4-O-coumaroylquinate (7), ${ }^{12}$ orcinol glucoside $(8)^{13,14}$ and 2,6-dimethoxy-benzioc acid (9), ${ }^{2}$ were also isolated from the Rhizomes of C. capitulata. Their structures were elucidated by spectral data and their comparison with literature values. Compounds 4-7 were isolated for the first time from this plant, and compound 7 was the first example isolated from the family.

Acknowledgments. This work was supported by the National
Natural Science Foundation of China (31070315), the International Foundation for Science (F/4340-1), and the Foundation of personnel developing of Anhui Province (2008Z020). The authors are grateful to the staff of the Modern Experiment Technology Center, Anhui University for the spectral data.

\section{References}

1. Lee, S. S.; Chang, W. L.; Chen, C. H. Tetrahedron Lett. 1996, 37 , 4405.

2. Chang, W. L.; Lee, S. S. Phytochemistry 1998, 49, 2133.

3. Li, N.; Chen, J. J.; Zhao, Y. X.; Zhou, J. J. Asian Nat. Prod. Res. 2005, 7, 189 .

4. Chang, W. L.; Chen, C. H.; Lee, S. S. J. Nat. Prod. 1999, 62, 734.

5. Chang, W. L.; Su, M. J.; Lee, S. S. J. Nat. Prod. 1997, 60, 76.

6. Cometa, M. F.; Palazzino, G.; Galeffi, C.; Palmery, M. Il Farmaco 2001, 56, 353.

7. Li, N.; Chen, J. J.; Zhou, J. J. Asian Nat. Prod. Res. 2005, 7, 279.

8. Wang, K. J.; Zhu, C. C.; Di, L.; Li, N.; Zhao, Y. X. Fitoterapia 2010, 81,869

9. Li, N.; Zhu, C. C.; Xiao, H. M.; Wang, K. J. Fitoterapia 2010, 81, 528

10. Wang, K. J.; Li, N. Arch. Pharm. Res. 2008, 31, 1313.

11. Zhu, C. C.; Wang, K. J.; Wang, Z. Y.; Li, N. Bull. Korean Chem. Soc. 2010, 31, 224.

12. Liu, Y. Z.; Yi, C. R.; Feng, W. S.; Xie, J. X. Chin. Tradit. Herbal. Drugs 1989, 20, 9.

13. Xu, J. P.; Dong, Q. Y. Chin. Tradit. Herbal. Drugs 1986, $17,8$.

14. Wu, Q.; Fu, D. X.; Hou, A. J.; Lei, G. Q.; Liu, Z. J.; Chen, J. K.; Zhou, T. S. Chem. Pharm. Bull. 2005, 53, 1065. 